\title{
Mycobacterium tuberculosis in a calf in West Texas: A rare pathology
}

\author{
Hawa Edriss MD, Melanie Lee MSN, Kenneth Nugent MD
}

\begin{abstract}
The transmission of Mycobacterium tuberculosis from humans to cattle is relatively uncommon and has not been reported in the United States. In the summer of 2018, a 5-month-old calf on a dairy farm in North West Texas failed a caudal fold test during routine screening. The animal was eventually euthanized, and a lymph node culture was positive for Mycobacterium tuberculosis, genotype G08735. Studies in Ethiopia have also reported that animals can become infected with Mycobacterium tuberculosis under certain conditions. This process likely depends on the methods used to manage livestock, the frequency of tuberculosis in adult populations, and the characteristics of the tuberculosis in these farm workers; its identification has important implications for workers and cattle.
\end{abstract}

Keywords: cattle, Mycobacterium tuberculosis, transmission

\section{INTRODUCTION}

Tuberculosis can infect both wild and domestic animals, including cattle. ${ }^{1,2}$ The prevalence of $M$. bovis in cattle is increasing in some countries, including Ethiopia, the United Kingdom, and Ireland, which has important health and economic consequences in countries like Ethiopia which has the largest livestock population in Africa. ${ }^{3}$ The mycobacterial species that infects cattle is mostly $M$. bovis and to a lesser extent $M$. caprae. Mycobacterial tuberculosis infection in cattle is a rare disease, seen in developing countries, and influenced by factors, such as close contact between farmers and cattle, inadequate infection control, and some traditional beliefs. ${ }^{2,4,5}$

\section{CASE}

In the summer of 2018, a 5-month-old calf on a dairy farm in Northwest Texas failed a caudal fold test

Corresponding author: Hawa Edriss

Contact Information: Edriss_ha07@yahoo.com

DOI: $10.12746 /$ swrccc.v8i33. $\overline{6} 43$ during routine screening. The animal was eventually euthanized, and a lymph node culture was positive for Mycobacterium tuberculosis, genotype G08735. The histology was negative. Investigation of the available dairy workers did not identify anyone with active pulmonary tuberculosis. However, one worker with cough and weight loss had left the farm approximately 2 weeks after the calf arrived. Three adults with pulmonary tuberculosis in this region had been diagnosed in 2017 and 2018 and had isolates with the same genotype.

\section{Discussion}

\section{EPIDEMIOLOGY}

The most common pathogens in mycobacterium complex are $M$. tuberculosis and $M$. bovis. Tuberculosis in animals is characterized by formation of granulomas, mostly in the lymph nodes of the head, lungs, pleura, intestines, liver, spleen, and peritoneum. ${ }^{6}$ Infection in cattle by $M$. bovis involves environmental factors, age, etc.; for example, the intestinal route of infection is more frequent in young calves due to ingestion of infected milk from tuberculous mastitis. However, this route is uncommon in the developed 
countries due to extensive eradication programs. In developed countries, the respiratory tract is the main source of disease transmission. On rare occasions, genital transmission occurs in cattle if genital organs become infected. ${ }^{7}$

Developing countries have a higher infectious disease burden in humans and animals due to the close physical contact between humans and animals, and the lack of disease control measures allows the transmission of the disease between animals and humans. ${ }^{5}$ It is well known that $M$. bovis can infect humans and causes zoonosis. ${ }^{8}$ The main sources of $M$. bovis transmission to human are through ingestion of raw milk in cattle with tuberculous mastitis and/or inhalation of aerosols from diseased animals. However, M. tuberculosis is mainly a human pathogen, and transmission to animals is very rare. ${ }^{1,2,9,10}$ Veterinarian studies indicate that M. tuberculosis infection in cattle occurs when cattle and humans have close and prolonged contact. ${ }^{11}$ The frequency of such transmission is unknown, but published reports suggest this frequency is less than $1 \% .^{12-15}$ In certain geographic locations, such as Ethiopia, Algeria, and Sudan, the frequency can approach $6 \%-7 \%{ }^{16}$ Transmission of $M$. tuberculosis to cattle can involve inhalation of aerosols from infected workers, the ingestion of grass contaminated with sputum, feces, and urine from infected patients, ${ }^{1,2,4}$ and direct human to animal contact through the tradition of spitting tobacco juice into the cattle's oral cavity in certain regions of Africa where it is believed to prevent parasitic infections and improve animal health. ${ }^{4,5}$

\section{Pathogenesis}

Observational studies indicate that infection with M. tuberculosis is much less aggressive in cattle than is infection with $M$. bovis. ${ }^{17}$ In fact, exposure to M. tuberculosis may transiently infect animals but not cause disease. Similarly, $M$. bovis is much less virulent in humans than $M$. tuberculosis. ${ }^{18}$ The exact pathogenesis, including disease transmission, sensitization, presence or absence of clinical manifestation, and the factors under which cattle become infected, is not well understood. ${ }^{3,19} M$. tuberculosis in cattle thought to cause a short period of infection and not progressive disease. The infected cattle have a positive reaction when tested with tuberculin, but the positivity disappears when the infectious source is cleared. Consequently, when a tuberculin-positive test is noted in previously tuberculin-negative cattle, the possibility of $M$. tuberculosis infection in farm workers should be considered.

Infected animals with $M$. tuberculosis represent accidental hosts. ${ }^{13}$ Actively infected humans with M. tuberculosis are the main source of transmission to animals. M. tuberculosis does not have a known animal reservoir host. ${ }^{13,20}$ In contrast, $M$. bovis can be maintained in a reservoir host in other species, such as buffalos, badgers, etc., from which infection is transmitted to the target host. ${ }^{6}$ In Ireland, cattle are considered the main source (reservoir) for maintaining infection. ${ }^{21}$

\section{Clinical studies}

Ameni studied tuberculosis transmission between farmers and cattle in 287 herds with 2,033 cattle in Ethiopia. ${ }^{5}$ The investigations in farmers included microbiological testing and molecular typing. The cattle underwent an intradermal tuberculin testing, microbiological examinations, post mortem examinations, and molecular typing The results indicated the prevalence of positive tuberculin tests in these herds was $9.4 \%$, and it was higher in herds owned by farmers with tuberculosis compared to herds owned by farmers without tuberculosis $(p<0.01)$. All mycobacteria isolated (141) from farmers were $M$. tuberculosis. Eleven out of sixteen isolates from cattle were non-tuberculosis mycobacteria (NTM), 3 isolates were $M$. bovis, and 2 were $M$. tuberculosis. Farmers with active pulmonary tuberculosis underwent clinical evaluations and had positive cultures in $97 \%$ (141/146). These $141 \mathrm{M}$. tuberculosis culture underwent characterization by spoligotyping; the most common patterns were Spoligo International Type Number (SIT) 149, SIT53, and SIT37. Molecular typing was also performed on cattle's isolates to identify the strain type. The $2 \mathrm{M}$. tuberculosis isolates from cattle were further characterized by spoligotyping, and the pattern of the isolates were SIT149 and SIT53 which matched the farmers' type. A severity scoring method using Vordermeier' $s$ system showed the average severity scores of the cattle infected with M. bovis, NTM, and M. tuberculosis were $5.5,2.1$, and 0.5 , respectively. ${ }^{22}$ 
These investigators suggested that the transmission of tuberculosis from farmers to cattle can infect cattle but rarely leads to active disease. ${ }^{5}$

In 2011, Ameni reported the characteristics of mycobacteria isolated from 52 cattle ( 30 from intensive production farm and 22 from grazing animals). Molecular typing of these isolates revealed 31 of these isolate were M. bovis, 6 M. tuberculosis, 6 M. avium species, and 9 other mycobacteria. ${ }^{23}$ All 30 isolates from the intensive production farm were $M$. bovis and were recovered from thoracic lymph nodes; most of the $M$. tuberculosis isolates were recovered from the mesenteric and retropharyngeal regions. The anatomic location may support the transmission via the traditional direct spitting tobacco juice into the cattle "mouth-to-mouth" in contrast to the $M$. bovis in which cattle had respiratory tract lesions and presumably a respiratory route of transmission between animals. Unfortunately, no M. tuberculosis samples were collected from workers on the farms in the study. However, prior DNA analysis identified that the 6 $M$. tuberculosis isolate types were previously found in humans in Ethiopia.

Ocepek reported that 3 animals with strongly reactive tuberculin tests ( 2 cows and one calf) were slaughtered in the Slovenian capital (Ljubljana). ${ }^{20}$ These 3 animals were clinically healthy except mildly enlarged lymph nodes. No acid-fast bacilli were detected during Ziehl-Neelsen staining. Histopathological examination of the mediastinal lymph nodes showed nonspecific findings; after 28-days colonies of $M$ tuberculosis grew on a Löwenstein-Jensen media from a 2-yearold cow. Investigation of the farmers identified a 59-year-old man who was diagnosed with a cavitary pulmonary tuberculosis in 1999. The M. tuberculosis isolates from the farmer and the cow were analyzed using an IS6110-based restriction fragment length polymorphism (RFLP) typing and had an identical RFLP pattern. Ocepek concluded that $M$. tuberculosis was transmitted from humans to cattle. ${ }^{20}$

\section{LABORATORY METHODS}

Molecular typing of $M$. tuberculosis subspecies using IS6110-based (RFLP) Restriction Fragment Length Polymorphism Typing is a widely used method for comparing the genetic relatedness of M. tuberculosis strains. It is used to determine suspected transmission and to identify previously unidentified links. It is based on the differences in fragment length and copy number of the insertion IS6110. Most isolates of $M$. tuberculosis contain between $0-20$ copies of the $1355 \mathrm{bp}$ IS6110. In most strains of $M$. tuberculosis, the arrangement of IS6110 in the genome appears stable over months to years. This analysis requires a large amount of DNA, long subculture (20-40 days), and technical complexity. ${ }^{25,26}$

Spacer oligonucleotide genotyping (Spoligotyping) is a PCR-based method for detection and typing of $M$. tuberculosis. It is a PCR amplification of highly polymorphic direct repeats (DR region) in $M$. tuberculosis. This method is less complex and has equal or better discriminatory power. In PCR analysis, variable numbers of tandem repeats of different genetic sequences, such as mycobacterial interspersed repetitive units (MIRUs), are measured; this method is a fast ( 1 day) alternative to the IS6110-based DNA typing and is becoming the preferred method for molecular typing for epidemiological or surveillance purposes. ${ }^{25,27}$

Whole genome sequencing is a new molecular method for the study of $M$. tuberculosis and is used for diagnosis, the detection of drug resistance, and typing of $M$. tuberculosis. It is a time consuming, requires highly trained personnel, but has a very high discriminatory power to identify transmission series. ${ }^{28,29}$

Current laboratory technology is adequate to characterize the epidemiology of tuberculosis transmission from animals to humans and vice versa. However, the studies will require relatively sophisticated technology and will require the storage of isolate for at least 2 years in an effort to identify clear transmission pathways.

\section{Conclusion}

We report an unusual situation in which a calf in West texas had infection with human Mycobacterium tuberculosis. The route of infection in this animal is uncertain. It likely related to direct transmission 
from a farm worker to either its mother or to the animal. State laboratories should perform a detailed molecular investigation of the specimens of slaughtered tuberculin-positive animals to identify cases of M. tuberculosis vs. M. bovis infections, and farmworkers need evaluation for latent or active tuberculosis. Prior reports suggest that transmission of tuberculosis from humans to cattle by respiratory system can infect the animals but rarely leads to active tuberculosis. Similarly, low transmission of $M$. bovis between cattle and farmers can occur.

Article citation: Edriss $\mathrm{H}$, Lee M, Nugent K. Mycobacterium tuberculosis in a calf in West Texas: a rare pathology. The Southwest Respiratory and Critical Care Chronicles 2020;8(33):63-67

From: Pulmonary and Critical Care Medicine (HE), Saint Joseph Hospital/KentuckyOne Health Group, Lexington, KY; Texas Department of State Health Services (ML), Lubbock, Texas; Department of Internal Medicine (KN), Texas Tech University Health Sciences Center, Lubbock, Texas

Submitted: $5 / 31 / 2019$

Accepted: $11 / 24 / 2020$

Reviewer: David Sotello MD

Conflicts of interest: none

This work is licensed under a Creative Commons

Attribution-ShareAlike 4.0 International License.

\section{REFERENCES}

1. Alexander KA, Pleydell E, Williams MC, et al. Mycobacterium tuberculosis: an emerging disease of free-ranging wildlife. Emerging Infect Dis 2002;8:598-601.

2. Michel AL, Venter L, Espie IW, et al. Mycobacterium tuberculosis infections in eight species at the National Zoological Gardens of South Africa, 1991-2001. J Zoo Wildl Med 2003;34:364-70.

3. Phillips CJ, Foster CR, Morris PA, et al. The transmission of Mycobacterium bovis infection to cattle. Res Vet Sci 2003; 74:1-15.

4. Ameni G, Amenu K, Tibbo, M. Bovine tuberculosis: Prevalence and risk factor assessment in cattle and cattle owners in Wuchale-Jida District, central Ethiopia. Int J Appl Res Vet Med 2003;1(1):17-26.
5. Ameni G, Tadesse $\mathrm{K}$, Hailu E, et al. Transmission of Mycobacterium tuberculosis between farmers and cattle in central Ethiopia. PLoS One 2013;8:e76891.

6. Pesciaroli M, Alvarez J, Boniotti MB, et al. Tuberculosis in domestic animal species. Res Veter Sci 2014;97:S78-S85.

7. Francis J. Route of infection in tuberculosis. Aust Vet J 1972; 48:578.

8. Cosivi O, Grange JM, Daborn CJ, et al. Zoonotic tuberculosis due to Mycobacterium bovis in developing countries. Emerg Infect Dis 1998;4:59-70.

9. Pavlik I, Yayo Ayele W, Parmova I, et al. Mycobacterium tuberculosis in animal and human populations in six Central European countries during 1990-1999. Vet Med-Czech 2003; 48:83-89.

10. LoBue PA, Enarson DA, Thoen CO. Tuberculosis in humans and animals: an overview. Int J Tuberc Lung Dis 2010;14: 1075-8.

11. Duma RJ. CRC Handbook Series in Zoonoses, James H. Steele, Ed in Chief, Section A: Bacterial, Rickettsial, and Mycotic Diseases. JAMA 1981;245:185.

12. Lesslie IW, Birn KJ. Mycobacterium avium infections in cattle and pigs in Great Britain. Tubercle 1970;51:446-51.

13. Thoen $\mathrm{CO}$, Karlson AG, Himes EM. Mycobacterial infections in animals. Clin Infect Dis 1981;3:960-72.

14. Pavlik I, Yayo Ayele W, Parmova I, et al. Incidence of bovine tuberculosis in cattle in seven Central European countries during the years 1990-1999. Veterinární Medicína 2012;47:45-51.

15. Schliesser T. [Presence and significance of mycobacteria in animals (proceedings)]. Zentralbl Bakteriol Orig A 1976;235:184-94].

16. Sulieman MS, Hamid ME. Identification of acid fast bacteria from caseous lesions in cattle in Sudan. J Veterinary Med Series B 2002;49:415-8.

17. Acha PN, Szyfres B. Zoonotic tuberculosis In: Zoonoses and communicable diseases common to man and animals, 3rd ed Vol I, Bacterioses and mycoses. Pan American Health Organization/World Health Organization, Washington. 2001. Pages 283-397.

18. Magnus K. Epidemiological basis of tuberculosis eradication. 3. Risk of pulmonary tuberculosis after human and bovine infection. Bull World Health Organ 1966;35:483-508.

19. Neill SD, Pollock JM, Bryson DB, et al. Pathogenesis of Mycobacterium bovis infection in cattle. Vet Microbiol 1994; 40:41-52.

20. Ocepek M, Pate M, Zolnir-Dovc M, et al. Transmission of Mycobacterium tuberculosis from human to cattle. J Clin Microbiol 2005;43:3555-7.

21. Neill SD, Hanna J, O’Brien JJ, et al. Transmission of tuberculosis from experimentally infected cattle to in-contact calves. Vet Rec 1989;124:269-71. 
22. Vordermeier HM, Chambers MA, Cockle PJ, et al. Correlation of ESAT-6-specific gamma interferon production with pathology in cattle following Mycobacterium bovis BCG vaccination against experimental bovine tuberculosis. Infect Immun 2002;70:3026-32.

23. Ameni G, Vordermeier M, Firdessa R, et al. Mycobacterium tuberculosis infection in grazing cattle in central Ethiopia. Vet J 2011;188:359-61.

24. Ameni G, Aseffa A, Engers H, et al. Cattle husbandry in Ethiopia is a predominant factor affecting the pathology of bovine tuberculosis and gamma interferon responses to mycobacterial antigens. Clin Vaccine Immunol 2006;13:1030-6.

25. Filliol I, Ferdinand S, Sola C, et al. Spoligotyping and IS6110-RFLP typing of Mycobacterium tuberculosis from French Guiana: a comparison of results with international databases underlines interregional transmission from neighboring countries. Res Microbiol 2002;153:81-8.
26. van Soolingen D, de Haas PE, Hermans PW, et al. Comparison of various repetitive DNA elements as genetic markers for strain differentiation and epidemiology of Mycobacterium tuberculosis. J Clin Microbiol 1993;31:1987-95.

27. Barlow RE, Gascoyne-Binzi DM, Gillespie SH, et al. Comparison of variable number tandem repeat and IS6110restriction fragment length polymorphism analyses for discrimination of high- and low-copy-number IS6110 Mycobacterium tuberculosis isolates. J Clin Microbiol 2001; 39:2453-7.

28. Satta G, Atzeni A, McHugh TD. Mycobacterium tuberculosis and whole genome sequencing: a practical guide and online tools available for the clinical microbiologist. Clin Microbiol Infect 2017;23:69-72.

29. van Soolingen D, Jajou R, Mulder A, et al. Whole genome sequencing as the ultimate tool to diagnose tuberculosis. Int J Mycobacteriol 2016;5 Suppl 1:S60-S1. 